Background Applying of stents for endovascular treatment of cerebral aneurysms in the acute period of intracranial hemorrhage remains a debatable topic at present time. In this work we reflected our experience in treatment of acute aneurysms,

Materials and Methods January 2011 through December 2019, 186 patients with 204 cerebral aneurysms were treated in our department in the acute period of hemorrhage: 27 patients had multiple aneurysms (2 to 5). Patients were 20 to 78 years old (47.2 years mean). Operations were performed in the period from 0 to 19 days after the rupture of the aneurysm.

Results The most frequent localization of aneurysms in our series is the supraclinoid part of the internal carotid artery $40.2 \%$, ACA aneurysms - $25.8 \%$, bifurcation of the middle cerebral artery - $17.5 \%$; posterior circulation was in $12.4 \%$ of cases.Coiling without assistance was used in $44.7 \%$ of aneurysms; $33.6 \%$ of aneurysms required the use of balloons; $21.5 \%$ of aneurysms required stent assistance.Stent implantation in the acute period of subarachnoid hemorrhage was used after transvenous administration of Eptifibatide (Integrilin); after the procedure patients received oral antiplatelet agents. No perioperative complications were reported in our series. General disability is $6.3 \%$, mortality $-2.1 \%$.

Conclusion Coiling with intracranial stents is effective for complex aneurysms with a wide neck in the acute period of intracranial hemorrhage, and it allows achieving a good result without increasing the risk of surgical treatment.

Disclosures V. Berestov: None. K. Orlov: None. N. Strelnikov: None. M. Demyanovskaya: None.

\section{E-219 UTILITY OF REPEAT ANGIOGRAPHY IN ANEURYSMAL- PATTERN ANGIOGRAPHICALLY NEGATIVE SUBARACHNOID HEMORRHAGE}

C Nesvick*, S Oushy, K Ravindran, L Rinaldo, P Kerezoudis, E Wijdicks, G Lanzino, A Rabinstein. Neurologic Surgery, Mayo Clinic, Rochester, MN

10.1136/neurintsurg-2020-SNIS.250

Background Some patients with aneurysmal-pattern subarachnoid hemorrhage (SAH) have a normal digital subtraction angiogram (DSA). Patients with angiographically negative subarachnoid hemorrhage (anSAH), including those with either perimesencephalic (panSAH) or aneurysmal-pattern hemorrhage (aanSAH, also known as diffuse anSAH), have an excellent prognosis if underlying vascular lesions are definitively excluded. Many centers perform a second DSA in these patients, but the rate of aneurysm detection on repeat DSA varies between studies, and the timing of this study is debated.

Methods All patients initially diagnosed with aanSAH after a screening DSA at a single tertiary neurovascular referral center January 2006 - April 2018 were included in this study. Patients with perimesencephalic-pattern, sulcal hemorrhage and traumatic SAH were excluded. A systematic review and metaanalysis of positive second DSA was performed on published case series of patients with aanSAH and at least two serial DSAs. The primary outcome for this analysis was pooled incidence using a binary random-effects model with studies weighted by size. I-squared statistics were calculated to assess heterogeneity.

Results Three patients (11.1\%) with aanSAH and multiple DSAs were found to have a cerebral aneurysm on repeat DSA at our institution. Each aneurysm was small (2 $\mathrm{mm}$ or smaller in maximum diameter). In a systematic review of 26 studies on the utility of repeat DSA for aanSAH, the pooled rate of positive second DSA was 10.4\% (95\% CI 7.3\% $13.5 \%, P<0.001)$, but substantial inter-study heterogeneity was observed $\left(\mathrm{I}^{2}=61.7 \%, P<0.001\right)$. Ten studies performed repeat DSA within two weeks of ictus, and two studies performed repeat DSA at least six weeks after ictus. Studies with early repeat DSA demonstrated a non-inferior aneurysm detection rate (pooled incidence $8.1 \%$ vs. $5.7 \%$ ). The pooled incidence of aanSAH across all non-traumatic $\mathrm{SAH}$ was $6.1 \% \quad\left(95 \% \quad\right.$ CI $\quad 4.7 \% \quad-\quad 7.5 \%, \quad P<0.001 ; \quad I^{2}=95.7 \%$, $P<0.001)$.

Conclusions About one in 10 patients with aanSAH have a ruptured cerebral aneurysm that is not detectable on initial DSA. These aneurysms tend to be very small and difficult to visualize using other imaging techniques. In patients with aneurysmal-pattern SAH and no detectable aneurysm, there is good evidence to perform a second DSA.

Disclosures C. Nesvick: None. S. Oushy: None. K. Ravindran: None. L. Rinaldo: None. P. Kerezoudis: None. E. Wijdicks: None. G. Lanzino: None. A. Rabinstein: None.
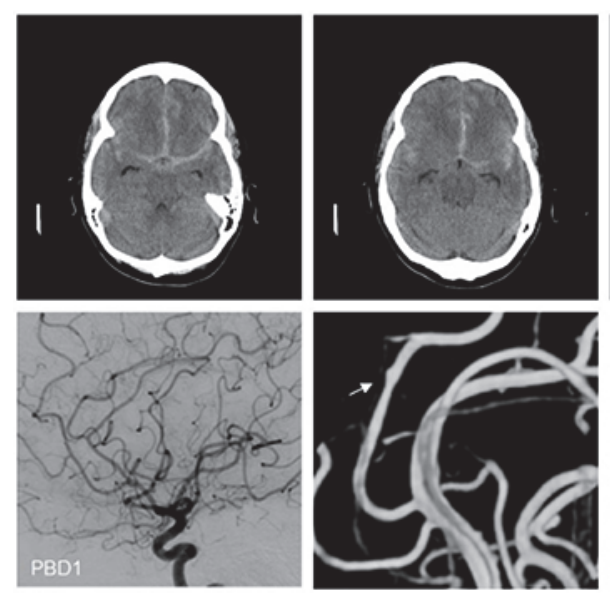
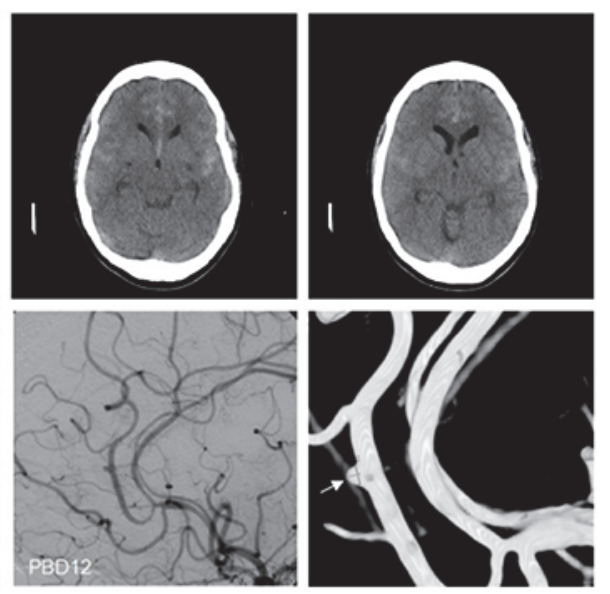

Abstract E-219 Figure 1 